\title{
RECURSIVE EVALUATION OF A FAMILY OF COMPOUND DISTRIBUTIONS*
}

\author{
Harry H. Panjer \\ University of Waterloo, Ontario, Canada
}

1. INTRODUCTION

Compound distributions such as the compound Poisson and the compound negative binomial are used extensivcly in the theory of risk to model the distribution of the total claims incurred in a fixed period of timc. The usual method of evaluating the distribution function requires the computation of many convolutions of the conditional distribution of the amount of a claim given that a claim has occurred. When the expected number of claims is large, the computation can become unwieldy even with modern large scale electronic computers.

In this paper, a recursive definition of the distribution of total claims is developed for a family of claim number distributions and arbitrary claim amount distributions. When the claim amount is discrete, the recursive definition can be used to compute the distribution of total claims without the use of convolutions. This can reduce the number of required computations by several orders of magnitude for sufficiently large portfolios.

Results for some specific distributions have been previously obtained using gencrating functions and Laplace transforms (sce PANJER (1980) including discussion). The simple algebraic proof of this paper yields all the previous results as special cases.

\section{THE FAMILY OF CLAIM NUMBER DISTRIBUTIONS}

Consider the family of claim number distributions satisfying the recursion

$$
p_{n}=p_{n-1}(a+b / n), \quad n=1,2,3 \ldots
$$

where $p_{n}$ denotes the probability that exactly $n$ claims occur in the fixed time interval. Members of this family are:

a) Poisson distribution:
1. $p_{n}=\frac{e^{-\lambda \lambda n}}{n !}, \quad n=0,1,2, \ldots$

* The author is grateful to the referee for pointing out an error in the original draft. This research was supported by the Natural Sciences and Enginecrmg Research Council of Canada. 
2. $p_{n} \mid p_{n-1}=\lambda / n, \quad p_{0}=e^{-\lambda}$

3. $a=0, b=\lambda$

b) Binomial distribution:

1. $p_{n}=\left(\begin{array}{l}N \\ n\end{array}\right) p^{n}(1-p)^{N-n}, \quad n=0,1,2, \ldots, N$

$p_{n}=0, \quad n=N+1, N+2, \ldots$

2. $p_{n} / p_{n-1}=(N-n+1) p\left((n(1-p)), \quad p_{0}=(1-p)^{N}\right.$

3. $a=-p /(1-p), \quad b=(N+1) p /(1-p)$

c) Negative binomial distribution:
1. $p_{n}=\left(\begin{array}{c}\alpha+n-1 \\ n\end{array}\right) p^{n}(1-p)^{\alpha}, \quad n=0,1,2, \ldots$
2. $p_{n} \mid p_{n-1}=(\alpha+n-1) p / n, \quad p_{0}=(1-p)^{\alpha}$
3. $a=p, \quad b=(\alpha-1) p$

d) Geometric distribution (Negative binomial with $\alpha=1$ ):
1. $p_{n}=(1-p) p^{n}, \quad n=0,1,2, \ldots$
2. $p_{n} / p_{n-1}=p, \quad p_{0}=1-p$
3. $a=p, \quad b=0$

SUNDT and JEWELL (1981) show that these are the only members of this family.

\section{THE RECURSIVE FORMULA}

Consider the compound distribution with distribution function

$$
\begin{aligned}
G(x) & =\sum_{n=1}^{\infty} p_{n} F^{* n}(x), & & x>0 \\
& =p_{0}, & & x=0
\end{aligned}
$$

for arbitrary claim amount distribution $F(x), x>0$. For notational convenience, assume that $F(x), x>0$, is of the continuous form. Corresponding results will be given for the discrete case. Then the density of total claims is

$$
\begin{aligned}
g(x) & =\sum_{n=1}^{\infty} p_{n} f^{* n}(x), & & x>0 \\
& =p_{0}, & x & =0
\end{aligned}
$$

when $f(x)$ is the density associated with $F(x)$.

In the theorem which follows, the following two relations will be used:

(I) $\quad \int_{0}^{x} f(y) f^{* n}(x-y) d y=f^{*(n+1)}(x), \quad n=1,2,3, \ldots$ 
(II) $\int_{0}^{\infty} y f(y) f^{* n}(x-y) / f^{*(n+1)}(x) d y=x /(n+1), \quad n=1,2,3, \ldots$

Relation (I) is the usual recursive definition of convolutions. The left side of relation (II) is the conditional mean of any element of a sum consisting of $n+1$ independent and identically distributed elements, given that the sum is exactly $x$. The mean is $x /(n+1)$ as a result of the symmetry in the elements of the sum. Relation (II) is used in Bühlmann and Gerber's discussion to PANJER (1980) to develop an alternate proof of the result described in that paper.

\section{Theorem}

For $p_{n}$ and $g(x)$ defined by (1) and (3) respectively, and $f(x)$ any distribution of the continuous type for $x>0$, the following recursion holds:

$$
g(x)=p_{1} f(x)+\int_{0}^{z}(a+b y / x) f(y) g(x-y) d y, \quad x>0 .
$$

\section{Proof}

Substituting (3) into the right side of (4) results in

$$
\begin{aligned}
p_{1} f(x)+\int_{0}^{x}(a+b y / x) f(y) & g(x-y) d y=p_{1} f(x)+\int_{0}^{x}(a+b y / x) f(y) \\
& =p_{1} f(x)+\sum_{n-1}^{\infty} p_{n} \int_{0}^{x}(a+b y \mid x) f(y) f^{* n}(x-y) d y \\
& =p_{1} f(x)+\sum_{n-1}^{\infty} p_{n}\{a+b /(n+1)\} f^{*(n+1)}(x) d y \\
& =p_{1} f(x)+\sum_{n-1}^{\infty} p_{n+1} f^{*(n+1)}(x) \quad \text { (from (I) and (II)) } \\
& \left.=p_{1} f(x)+\sum_{n-3}^{\infty} p_{n} f^{* n}(x) \quad \text { (since } f^{* 1}(x)=f(x)\right) \\
& =\sum_{n-1}^{\infty} p_{n} f^{* n}(x) \quad \text { Q.E.D. } \\
& =g(x), \quad \text { the required result. } \quad \text {. }
\end{aligned}
$$

If the claim amount distribution is discrete and defined on the positive integers, the corresponding recursive definition of total claims is

$$
g_{i}=\sum_{i=1}(a+b j / i) f_{j} g_{i-j}, \quad i=1,2,3, \ldots
$$


with $g_{0}=p_{0}$; whereas the usual form is

$$
g_{i}=\sum_{n=0}^{i} p_{n} f_{i}^{*} n, \quad i=0,1,2,3, \ldots
$$

The number of computations required to obtain $g_{i}$ is of order $i^{2}$ for formula (6) and of order $i$ for formula (5). Hence, for large values of $i$, the reduction in computations is dramatic.

\section{RESULTS FOR SPECIAL CASES}

The recursive definitions of the density of total claims for the four distributions considered in Section 2 are given below:

Continuous Model

$g(x)$
Discrete Model

$g_{i}$

a) Poisson

$$
\lambda e^{-\lambda} f(x)+\frac{\lambda}{x} \int_{0}^{x} y f(y) g(x-y) d y \quad \frac{\lambda}{i} \sum_{j=1}^{1} j f_{j} g_{i-j}
$$

b) Binomial

$$
\begin{aligned}
& \frac{p}{1-p}\left[N(1-p)^{N} f(x)+\int_{0}^{x}\{(N+1) y / x-1\} f(y) g(x-y) d y\right] \\
& \frac{p}{1-p} \sum_{i=1}^{i}\{(N+1) j / i-1\} f_{j} g_{i-j}
\end{aligned}
$$

c) Negative binomial

$$
\begin{array}{r}
p\left[\alpha(1-p)^{\alpha} f(x)+\int_{0}^{x}\{1+(\alpha-1) y / x\} f(y) g(x-y) d y\right] \\
p \sum_{j=1}^{1}\{1+(\alpha-1) j \mid i\} f_{j} g_{i-j}
\end{array}
$$

d) Geometric

$$
p\left[(1-p) f(x)+\int_{0}^{\pi} f(y) g(x-y) d y\right] \quad p \sum_{i=1}^{i} f_{1} g_{i-3}
$$

The recursion for the compound Poisson distribution with discrete claim amount distribution was originally given by ADELSON (1966) in an inventory problem. He used generating functions to obtain his result. 
SUNDT and JEWELL (1981) generalize the results of the present paper. They obtain results for a more general recursion than (1) and obtain results for the case of possibly negative claims.

\section{REFERENCES}

Adelson, R. M. (1966). Compound Poisson Distributions, Operations Research Quarterly, $17,73-75$.

Panjer, H. H. (1980). The Aggregate Claims Distribution and Stop-Loss Reinsurance, Trans. of the Society of Actuaries, XXXII.

Sundt, B, and JEwell, W. S. (1981). Further Results on Recursive Evaluation of Compound Distributions. Astin Bulletin 12 . 